culture, Tuan describes how the rainforest environment of the pygmy with its lack of horizon and landmarks, without pattern, has resulted in a world view in which the sense of distance and perspective is "distorted" and a sense of time is curtailed.

But to dip into the book, despite the inherent interest of every page, is not to do it justice because it must be cmphasised that Tuan has composed a coherent and systematic thesis, analysing those innate aspects of our values and perceptions which we seem to share with other life forms, from those which mark us as individuals, or as members of a certain culture or subgroup. Possibly, and this might be taken as an anticipation rather than an omission from an otherwise comprehensive work, Yi-Fu Tuan's thesis could have led into a deeper discussion of the evolving nature and complexity of contemporary society in relation to concepts such as "postindustrial society" and "future shock", but perhaps this is for another volume. What he has achieved is to extend a way of looking at the natural and physical environment which demonstrates and calls for an appreciation of our own and other people's values and way of life at all levels of planning. The book is inexpensive and well indexed and should provide a useful and thought provoking basic text for both the casual reader and university courses dealing with urban geography, man in relation to the environment or the humanities.

H. S. D. Cole

\section{Acoustic orientation}

Echolocation in Animals. By E. S. Airapet'yants and A. I. Konstantinov. (Translated from Russian.) Pp. vi+ 309. (Academy of Sciences of the U.S.S.R. Joint Scientific Council on Physiology of Man and Animals.) (Israel Program of Scientific Translations: Jerusalem; Wiley: Chichester; January 1974.) $£ 10.50$.

THis book was first published in the Soviet Union in 1970 . It reviews Russian literature up to 1969 and Western literature up to 1968 so that many important discoveries made within the past five years are not covered. The major part of the book is devoted to studies on bats. This includes sections on the biology of bats, the nature of sound waves and methods of studying bat sounds. The signals emitted by bats are described and methods of sound production and radiation are discussed. Studies on both the auditory system of bats and their echo location ability are dealt with in some detail. Echo location in birds ard small mammals is also discussed, but very briefly. The chapters on Cetacea include descriptions of their biology, mechanisms of sound production and reception, a review of the different signals emitted by these animals and a consideration of their echo location performance. The final chapter of the book is devoted to echo location in pinnipedes.

The main value of the book is that it draws together much recent Russian literature on echo location. Russian work is described in some detail, particularly in the sections on hearing in bats and on the echo location abilities of both bats and dolphins. Although this is useful for non-Russian readers, it often results in considerable imbalance in the treatment of different topics; a more comprehensive treatment of some topics would have been useful. For example, the authors describe their own equipment to illustrate methods of studying bat signals. But this excludes a consideration of equipment used by other workers, such as portable bat detectors and sound spectrographs, both of which are important in the study of bat signals. Displays from the latter, taken from the literature, are presented later in the book with no explanation and occasionally without adequate labelling.

The authors express the hope that the book will appeal to readers of many professions, but very little help is given to interdisciplinary readers or to newcomers to the subject. For instance the theoretical considerations of various aspects of echo location are often difficult to follow and many of the equations are given without any hint of derivation or a source reference. Also, descriptions of the anatomy of the larynx and the auditory pathways are given as a string of latin names, without adequate explanations or diagrams. The absence of an index is also a great drawback and makes the book difficult to use as a reference text.

On the whole the English translation from the Russian is very readable but there is some awkwardness of expression in which the exact meaning is often not clear. Some apparently literal translations have been given, for example 'humeral girdle' and 'cathode repeater' when these could have been fairly easily replaced by their more commonly accepted equivalents, here 'pectoral girdle' and 'cathode follower' respectively.

Some points of production are irksome. The latin names of species and genera are not italicised (this is a reversal from the Russian text) and the references given in the text and in the bibliography are not always accurate or consistent.

Because the literature on echo location is expanding so rapidly, a new comprehensive, critical review would be welcome. This book is disappointing both in the treatment of the material and in its presentation and does not fulfil the requirements of such a review. But because of its account of Russian work, the book will be a useful, though expensive, addition to literature on bioacoustics.

Gillian D. Sales

\section{Plant ecology in Africa}

East African Vegetation. By E.M. Lind and M. E. S. Morrison. Pp. xvii +257. (Longman: London, April 1974.) £6.

THE appearance of this work will be hailed by those interested in tropical Africa as an ecological milestone, for in spite of excellent herbaria and taxonomic works, biologists have had to work in these regions without the aid of a gencral description of the vegetation. Indeed in many cases, large mammal research schemes have been devised without reference to botanical studies.

The authors have avoided the difficulties of describing vegetation with long lists of plant names by referring to dominant species and community structure, and relating this information to details of microclimate, water relations, soils and productivity. Additionally, they have succeeded in relating botanical information to simple descriptions of animal communities.

Vegetation types are dealt with in considerable detail and include forest habitats, rangelands (grassland and wooded grassland), inland aquatic vegetation, coastal vegetation (including intertidal and mangrove swamps), and high altitude vegetation. Considering the difficulties of describing local conditions existing at altitudes ranging from sea level to 19,000 foot, the authors have achieved a good balance between purely descriptive material and broader ecological information.

Part two discusses the influence of water availability and temperature on plant form, structure and growth followed by a short chapter on soils: the description of catenary soil associations and their vegetation types is particularly valuable.

Alan Hamilton contributes a useful chapter on vegetational history, in which he examines evidence derived from macro fossils, pollen analysis and plant geography. Of particular interest is the author's comparison of the apparently impoverished African forests with those of Malaysia and South America. He concludes that the flora of these areas have been depauperated at a time when the climate was very dry.

This valuable addition to the tropical literature will be of great value to research workers, teachers and students at all levels, and with luck will provide a new impetus for field botanical studies in Afrioa. Malcolm Cor 